\title{
AN UNUSUAL CAUSE OF EMPYEMA THORACIS
}

A 45-year- old male, manual labourer, presented with a 3-month history of cough with moderate expectoration, intermittent fever and progressive breathlessness. There was no history of chest pain or haemoptysis. There was a history of loss of appetite and loss of weight of 5 kilograms over the past 3 months. He had been treated for pulmonary tuberculosis two years earlier with 6 months of regular Category 1 anti-tuberculous drugs under the DOTS program. He was not a diabetic and there was no history to suggest an underlying immunocompromised state. There was no past history of thoracocentesis or intercostal chest tube insertion. He was a chronic smoker for 20 years and an occasional consumer of alcohol.

On examination he was thinly built and afebrile. $\mathrm{He}$ had pallor and clubbing. Examination of the 
respiratory system revealed the presence of a left sided hydropneumothorax. The rest of the systemic examination was normal.

Investigations revealed a hemoglobin of 8.9 gm\%, total WBC count of 11,800 cells/ cu $\mathrm{mm}$ with normal differential count, normal plasma glucose levels and renal functions. Liver function tests were normal except for a serum albumin of $2.4 \mathrm{~g} \%$. Serology for HIV, hepatitis $B$ and hepatitis $C$ were all negative. Chest X-ray showed evidence of a left sided hydropneumothorax [Figure 1]. Three sputum samples were negative for acid fast bacilli. Pleural fluid analysis revealed the presence of foul smelling pus which showed septate fungal hyphae on smear and subsequently grew Aspergillus terreus on culture. Pleural biopsy was also done which showed the same organism on smear and culture. Histopathology showed acute inflammatory exudate and fungal filaments suggestive of Aspergillus species. Routine bacterial and mycobacterial cultures were sterile. Ultrasound abdomen was normal. CT scan of the thorax showed moderate loculated hydropneumothorax [Figure 2] on

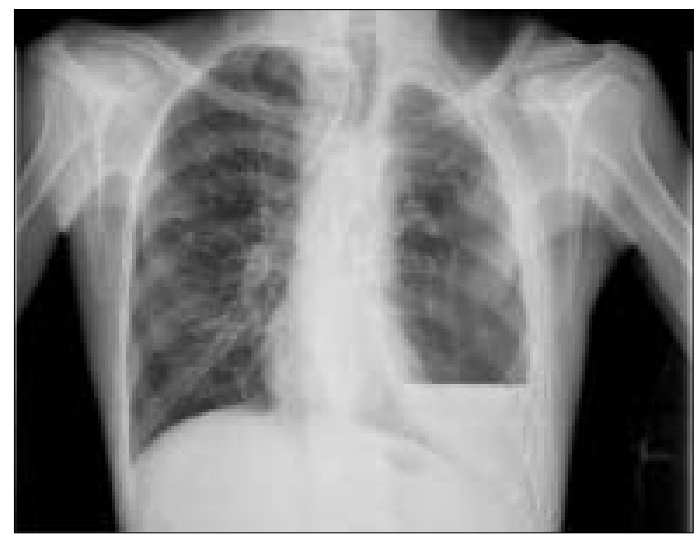

Figure 1: Chest $X$-ray showing left sided Hydropneumothorax the left side with compensatory hypertrophy on the right side, with foci of bronchiectasis and calcification bilaterally and small hilar and mediastinal nodes.

He was treated with intercostal drainage, Amphotericin B at a daily dose of $1 \mathrm{mg} / \mathrm{kg}$ to complete a total of $1.5 \mathrm{gm}$ of Amphotericin B. As the underlying lung had not significantly expanded and there was a persistent air leak he was advised left lung decortication surgery. His symptoms of fever, cough and breathlessness had markedly improved with the above therapy.

Aspergillus may cause a broad spectrum of disease in the human host, ranging from hypersensitivity reactions to direct angioinvasion. Aspergillus primarily affects the lungs, causing four main syndromes, including allergic bronchopulmonary aspergillosis (ABPA), chronic necrotizing Aspergillus pneumonia, aspergilloma, and invasive aspergillosis. The commonest isolated species are Aspergillus fumigatus and Aspergillus flavus. ${ }^{[1]}$ Aspergillus terreus infections are

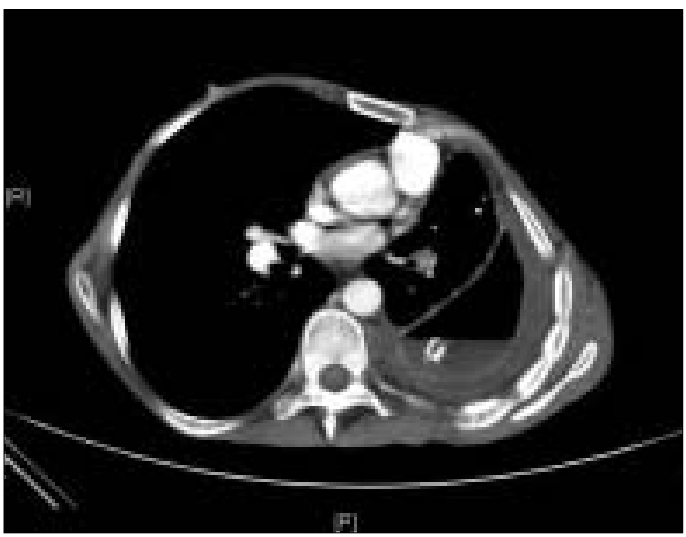

Figure 2: CT Thorax showing a loculated left sided hydropneumothorax 
uncommon, the frequency ranging between $3-12.5 \%$.

Pleural aspergillosis is not characteristically associated with pulmonary aspergillosis in either its allergic, bronchopneumonic, or intracavitary forms ${ }^{[2]}$ It has also not been described in the opportunistic setting. In a series of 98 such patients mainly with lymphoreticular or hematopoietic malignancies, the pleura was not involved, although the lungs were involved in over $90 \%$ and in many the disease was invasive and widespread. ${ }^{[3]}$

Among the described cases of pleural aspergillosis, etiologic factors include: pre-existing pulmonary tuberculosis $87 \%$; bronchopleural fistula - $74 \%$; pleural drainage - 56\%; and lung resection $17 \% .{ }^{[2]}$ In most patients multiple antibiotic therapy was administered. The disease occurred most commonly in patients with an established empyema and a bronchopleural or pleurocutaneous fistula. The diagnosis of pleural aspergillosis is established by demonstration of the organism in pleural discharge or by pleural biopsy. ${ }^{[2]}$

Early administration of antifungal agents and pleural drainage are thought to be helpful in improving the outcome for patients with pleural aspergillosis. ${ }^{[4]}$ Amphotericin $B$ is the main drug for the treatment of most fungal infections..However the major limitation of Amphotericin B is its toxicity (nephrotoxicity, phlebitis, hypokalaemia, hypomagnesaemia, and anaemia) seen in nearly $80 \%$ of patients. ${ }^{[5]}$ Liposomal Amphotericin B offers the potential of a less toxic intravenous alternative to conventional Amphotericin B. Certain centers also advocate the use of intra pleural instillation of Amphotericin B with varying results. ${ }^{[2]}$

Voriconazole is a broad-spectrum triazole that is active against aspergillus species. Recent studies have shown that treatment with Voriconazole is better than treatment with Amphotericin B for invasive Aspergillosis. Voriconazole was better tolerated than amphotericin B, with fewer drugrelated adverse events. ${ }^{[2]}$

RAJIV K. KARTHIK, T. D. SUDARSANAM Department of Medicine Unit 2, Christian Medical College and Hospital, Vellore-632 004, India

Corresponding:
Dr. Rajiv Karthik, Department of Medicine Unit 2,
Christian Medical College and Hospital,
Vellore -632004 , India.
E-mail: rajiv@ cmcvellore.ac.in

\section{REFERENCES}

1. Steinbach WJ, Benjamin DK Jr, Kontoyiannis DP, Perfect JR, Lutsar I, Marr KA, et al. Infections due to Aspergillus terreus: A multicenter retrospective analysis of 83 cases. Clin Infect Dis 2004;39: 192-8.

2. Meredith HC, Cogan BM, Mclaulin B. Pleural Aspergillosis. AJR Am J Roentgenol 1978;130:164-6.

3. Young RC, Bennett JE, Vogel CL, Carbone PP, DeVita VT. Aspergillosis. Medicine 1970;49: 147-73.

4. Ko SC, Chen KY, Hsueh PR, Luh KT, Yang PC. Fungal empyema thoracis: An emerging clinical entity. Chest 2000;117:1672-8.

5. Herbrecht R, Denning DW, Patterson TF, Bennett JE, Greene RE, Oestmann JW, et al. Randomised comparison of voriconazole and amphotericin $B$ in primary therapy of invasive aspergillosis. $\mathrm{N}$ Engl J Med 2002;347:408-15.

DOI: 10.4103/0019-5359.49081 